\title{
Efecto de la gonadotrofina coriónica equina y del inseminador sobre la preñez en vacas con cría en inseminación artificial a tiempo fijo
}

\author{
Aba, M. ; Chayer, R. ${ }^{2}$; Uslenghi, G. ${ }^{3,4}$; González-Chaves, S. ${ }^{2}$; Callejas, S. ${ }^{4}$ \\ ${ }^{1}$ Tesinista Facultad de Ciencias Veterinarias UNCPBA, ${ }^{2}$ Asesor actividad privada. Grupo CONPAS, ${ }^{3}$ Becario \\ Posgrado CONICET, ${ }^{4}$ Área de Reproducción, Facultad de Ciencias Veterinarias UNCPBA, Paraje Arroyo \\ Seco s/n (7000) Tandil (Argentina). Tel./Fax: 0249-4439850. E-mail: callejas@vet.unicen.edu.ar.
}

\begin{abstract}
Resumen
Aba, M.; Chayer, R.; Uslenghi, G.; González-Chaves, S.; Callejas, S.: Efecto de la gonadotrofina coriónica equina y del inseminador sobre la preñez en vacas con cría en inseminación artificial a tiempo fijo. Rev vet. 24: 1, 25-28, 2013. Los objetivos de este trabajo fueron evaluar el uso de la gonadotrofina coriónica equina (eCG) administrada al retirar un dispositivo intravaginal con progesterona (DISP) y el efecto del inseminador, en vacas con cría, sin cuerpo lúteo. El día 0 , se seleccionaron mediante ecografía vacas sin cuerpo lúteo $(\mathrm{n}=223)$, se determinó la condición corporal $(\mathrm{CC})$ y se colocó un DISP $(1,2 \mathrm{~g}$ de progesterona, EMEFUR ${ }^{\circledR}$, Merial) más $2 \mathrm{mg}$ de benzoato de estradiol (BE; EMEFUR®, Merial) im. El día 8 se retiró el DISP, se administró (im) $0,15 \mathrm{mg}$ de D (+) Cloprostenol (EMEFUR ${ }^{\circledR}$, Laboratorio Merial) y los animales se distribuyeron aleatoriamente teniendo en cuenta la CC (=4 o 5-6) y la estructura ovárica predominante (Folículos $-\mathrm{F}-<\mathrm{o} \geq 10 \mathrm{~mm}$ ) para recibir o no (Control) 400 UI de eCG (Novormon, Syntex SA). El día 9 se aplicó $1 \mathrm{mg}$ de BE im y los animales fueron inseminados a tiempo fijo (IATF) a las 52-56 h post-retiro del DISP. El diagnóstico de gestación se realizó mediante ecografía a los 28 días post IATF. Se evaluó el porcentaje de preñez según Tratamiento (eCG y control), CC (4 y 5-6), estructura ovárica predominante (EO, folículos $-\mathrm{F}-<10 \mathrm{~mm}$ y $\mathrm{F} \geq 10 \mathrm{~mm}$ ), Inseminador (A y B) y sus interacciones. Para las estadísticas se utilizó el PROC CATMOD del SAS, fijando un nivel de confianza del $95 \%(\alpha=0,05)$. No se observaron diferencias significativas para Tratamiento (eCG: 48,6\%; Control: 45,5\%), CC (4: 37,0\%; 5-6: 49,7\%) y EO (F<10mm: 45,8\%; F $\geq 10 \mathrm{~mm}: 47,6 \%)$ $(\mathrm{p}>0,05)$. Hubo efecto del Inseminador (A: $53,8 \%$; B: $25,0 \% ; \mathrm{p}<0,05$ ). Se concluye que la administración de eCG al final de un tratamiento para IATF en vacas con cría de condición corporal de 4 a 6 y folículos como estructura ovárica predominante, no mejora el porcentaje de preñez. Por el contrario, el técnico inseminador es un factor importante que afecta la eficiencia reproductiva.
\end{abstract}

Palabras clave: vacas con cría, gonadotrofina coriónica equina, inseminador, progesterona, inseminación artificial a tiempo fijo, preñez.

\begin{abstract}
Aba, M.; Chayer, R.; Uslenghi, G.; González-Chaves, S.; Callejas, S.: Effect of chorionic gonadotropin and inseminator on pregnancy in suckling beef cows at fixed-time artificial insemination. Rev vet. 24: 1, 25-28, 2013. The objectives were to evaluate the effects of equine chorionic gonadotropin $(\mathrm{eCG})$ at withdrawal of a progesterone intravaginal device (P4 device) and the inseminator on suckling beef cows without corpus luteum. On day 0, cows without corpus luteum were selected $(\mathrm{n}=223)$, body score condition $(\mathrm{BSC})$ were measured and cows received a $\mathrm{P} 4$ device (1.2 $\mathrm{g}$ of progesterone, EMEFUR $\AA$, Merial) and $2 \mathrm{mg}$ of estradiol benzoate (EB; EMEFUR ${ }^{\circledR}$, Merial) im. On day 8, P4 device were removed, $0.15 \mathrm{mg}$ of D (+) Cloprostenol (EMEFUR ${ }^{\circledR}$, Merial) im was administered and the cows were randomized into two groups to receive or not (Control) 400 IU of eCG (Novormon, Syntex), considering BSC ( $=4$ or 5-6) and dominant ovarian structure (DOS: Follicles $-\mathrm{F}-<$ or $\geq 10 \mathrm{~mm}$ ). On day 9, $1 \mathrm{mg}$ of EB was injected im and all cows were inseminated at a fixed-time (FTAI), 52-56 $\mathrm{h}$ after device withdrawal. Pregnancy diagnosis was performed by ultrasonography on day 28 after FTAI. The effect of treatment (eCG and control), BSC (4 and 5-6), DOS (F<10 $\mathrm{mm}$ and $\mathrm{F} \geq 10 \mathrm{~mm}$ ), inseminator ( $\mathrm{A}$ and $\mathrm{B}$ ) and their interactions upon pregnancy rate at FTAI were evaluated. Variables were analyzed using PROC CATMOD of SAS, significance was indicated by a probability of $<0.05$. There were no effect of treatments (eCG: $48.6 \%$;
\end{abstract}

Recibido: 30 abril 2013 / Aceptado: 31 mayo 2013 


\begin{abstract}
Control: 45.5\%), BSC (4: 37.0\%; 5-6: 49.7\%) and DOS ( $\mathrm{F}<10 \mathrm{~mm}: 45.8 \%$; $\mathrm{F} \geq 10 \mathrm{~mm}: 47.6 \%)$ $(\mathrm{p}>0.05)$. There was an effect of the inseminator (A: 53.8\%; B: 25.0\%; $<<0.05$ ). In conclusion, administration of eCG at the end of a FTAI treatment did not improve pregnancy rate in cows with a BSC from 4 to 6 and follicles as the dominant ovarian structure. On the other hand, the inseminator experience is an important factor that affects the reproductive efficiency.
\end{abstract}

Key words: suckling beef cows, equine chorionic gonadotropin, inseminator, progesterone, fixed-time artificial insemination, pregnancy rate.

\section{INTRODUCCIÓN}

El control farmacológico del ciclo estral facilita la implementación de la inseminación artificial en los rodeos de cría, con la consiguiente mejora genética y productiva que esto implica. Para evitar los problemas de la detección de celos, se han desarrollado tratamientos de sincronización de la ovulación que permiten inseminar un gran número de animales en un período de tiempo establecido, conociendo a esta técnica como Inseminación Artificial a Tiempo Fijo (IATF) ${ }^{2,8}$.

El tratamiento más difundido es el uso de dispositivos intravaginales con progesterona (DISP) durante 8 días, combinado con benzoato de estradiol (BE) al momento de colocar los dispositivos (para inducir una nueva onda de crecimiento folicular ${ }^{3,4,5}$ ) y un análogo sintético de la prostaglandina $\mathrm{F}_{2} \alpha$ al retiro del mismo (lisis del cuerpo lúteo ${ }^{3,9}$ ). Veinticuatro horas después se administra nuevamente BE para sincronizar las ovulaciones, de esta manera se puede realizar la IATF a las $52-56 \mathrm{~h}$ de retirado el dispositivo ${ }^{3}$. Adicionalmente, el tratamiento descrito permite inducir actividad sexual cíclica en animales en anestro ${ }^{14}$.

En rodeos de cría, el amamantamiento y el balance energético negativo son dos de los factores más importantes que incrementan el período de anestro post parto, debido a su efecto negativo sobre la frecuencia de pulsos de LH y el desarrollo folicular ${ }^{13}$. El tamaño del folículo dominante al final de un protocolo de sincronización es un factor que afecta la ovulación -en cantidad y en calidad- y el porcentaje de preñez ${ }^{13}$. Así, por ejemplo, la ovulación de un folículo dominante pequeño, y presumiblemente inmaduro fisiológicamente, reduce los porcentajes de preñez ${ }^{7,11,16} \mathrm{e}$ incrementa las pérdidas por mortalidad embrionaria o fetal ${ }^{11}$.

Teniendo en cuenta que existen tratamientos hormonales que directamente estimulan el desarrollo folicular, se puede determinar que la combinación de éstos con un tratamiento de sincronización de celos pueden mejorar los porcentajes de preñez. En este sentido, la gonadotrofina coriónica equina (eCG) ha sido utilizada en combinación con dispositivos intravaginales con progesterona con el objetivo de estimular el crecimiento folicular ${ }^{13}$.

Otro factor a tener en cuenta como determinante de la eficiencia en la implementación de los programas de IATF es la experiencia del técnico inseminador. En una reciente investigación se comunicaron hasta 10 puntos de diferencia numérica en la fertilidad sin obtener diferencias estadísticas, y se recalcó la necesidad de realizar más estudios en la temática ${ }^{1}$.

En función de lo planteado precedentemente, el objetivo del presente trabajo fue evaluar el efecto de la eCG sobre el porcentaje de preñez que se obtiene luego de realizar una IATF, en vacas con cría sin cuerpo lúteo (CL) al momento de iniciar el tratamiento. Como objetivo secundario, se planteó evaluar el efecto de dos técnicos inseminadores.

\section{MATERIAL Y MÉTODOS}

Lugar. El trabajo se realizó en un establecimiento comercial ubicado en el Partido de Las Flores, Provincia de Buenos Aires, Argentina.

Animales. Se utilizaron 250 vacas Bos taurus con cría al pie, con una condición corporal promedio de 4,9 90,5 (escala de 1 a 9) y 45 días como mínimo de período post parto. El 10,8\% de los animales tenía un $\mathrm{CL}$, lo cual indica que la mayor proporción del rodeo se encontraba en anestro. Estos animales no fueron utilizados en el trabajo.

Alimentación. Los animales recibieron alimentación pastoril sobre campo natural, sin suplementación, teniendo libre acceso al agua.

Tratamientos. En el día 0, las vacas fueron examinadas mediante ultrasonografía (Mindray DP-6600 Vet, equipado con un transductor de multifrecuencia de $5,0 \mathrm{MHz}$ ) para identificar la estructura ovárica predominante (folículos -F- o CL) y recibieron un dispositivo intravaginal con $1,2 \mathrm{~g}$ de progesterona (EMEFUR $\AA$, Laboratorio Merial), más $2 \mathrm{mg}$ de BE (Benzoato de estradiol EMEFUR $\AA$, Laboratorio Merial) (im). En el día 8 se retiró el dispositivo y se administró (im) $0,15 \mathrm{mg}$ de D (+) Cloprostenol (EMEFUR ${ }^{\circledR}$, Laboratorio Merial). Los animales que no presentaban CL ( $\mathrm{n}=223)$, fueron divididos aleatoriamente en dos grupos teniendo en cuenta la condición corporal $(=4$ o 5-6) y la estructura ovárica predominante $(\mathrm{F}<\mathrm{o} \geq 10 \mathrm{~mm})$ para recibir o no (Control) 400 UI de eCG (Novormon, Syntex SA). En el día 9 los animales recibieron $1 \mathrm{mg}$ de BE (im) y fueron inseminados a tiempo fijo a las 52-56 h de retirado el dispositivo intravaginal. Para la IATF se utilizó semen congelado/descongelado en pajuelas de $0,5 \mathrm{ml}$ proveniente de un toro de probada fertilidad, cuyo análisis de laboratorio fue: motilidad progresiva a las horas 0 y $2,40 \%$ y $35 \%$, vigor: 3 en ambas lecturas, morfo- 
logía: 88\% normales, número de espermatozoides con motilidad progresiva: mayor a 10.000 .000 por pajuela.

Diagnóstico de gestación. Se realizó mediante ultrasonografía (Mindray DP-6600 Vet, equipado con un transductor de multifrecuencia de 5,0 MHz.) a los 28 días post inseminación, para evaluar el porcentaje de preñez a la IATF.

Análisis estadístico. Se evaluó el efecto del tratamiento (eCG y control), de la condición corporal al inicio (4 y 5-6); de la estructura ovárica predominante ( $\mathrm{F}<10 \mathrm{~mm}$ y $\mathrm{F} \geq 10 \mathrm{~mm}$ ), del inseminador (A y B) y de sus interacciones, sobre el porcentaje de preñez. Se utilizó el PROC CATMOD del SAS ${ }^{15}$, fijándose un nivel de confianza del $95 \%(\alpha=0,05)$.

\section{RESULTADOS}

No se observaron efectos del tratamiento, de la condición corporal, de la estructura ovárica predominante y de sus interacciones, sobre el porcentaje de preñez a la IATF ( $\mathrm{p}>0,05$; Tabla 1). Por el contrario se observó un efecto significativo del inseminador (A: 53,8\%; B: $25,0 \% ; \mathrm{p}<0,05)$.

\section{DISCUSIÓN}

El porcentaje de preñez observado en el presente trabajo $(47,1 \%)$ se encuentra dentro del rango citado en la bibliografía, que varía de $23,6 \%$ a $74,4 \%{ }^{4,6}$. Otros investigadores reportaron que el tratamiento con 400 UI de eCG en vacas Bos indicus con cría aumenta el porcentaje de preñez a la IATF respecto de los animales que no recibieron el tratamiento hormonal ${ }^{13}$. Los resultados del presente trabajo no coinciden con esto, ya que no se observaron diferencias entre las vacas que recibieron o no eCG a pesar de estar en anestro post parto. Tal vez, ello puede deberse a la diferente duración del anestro post parto en los animales Bos indicus respecto de Bos taurus ${ }^{10}$.

No obstante, el $65,6 \%$ de las vacas del rodeo $(164 / 250)$ tenían un folículo mayor o igual a $10 \mathrm{~mm}$, lo que indicaría que estaban en un anestro superficial y tal vez no habrían necesitado del estímulo hormonal de la eCG para desarrollar el folículo dominante al momento de retirar el dispositivo intravaginal. Futuros trabajos deberán caracterizar la respuesta a la eCG en rodeos con diferentes porcentajes de vacas con folículos menores a $10 \mathrm{~mm}$ de diámetro.

Con respecto a la estructura ovárica, otros autores informaron similares porcentajes de preñez entre animales que tuvieron folículos $<\mathrm{o} \geq$ a $10 \mathrm{~mm}$ al momento de colocar los dispositivos intravaginales ${ }^{12}$, lo cual coincide con lo observado en el presente trabajo. Además, estos autores observaron que los animales que tenían folículos menores a $10 \mathrm{~mm}$ se preñaron en menor proporción que aquellos que tenían un cuerpo lúteo. En el presente trabajo los animales que tuvieron un cuerpo lúteo fueron excluidos del diseño experimental; no obstante, éstos $(n=27)$ tuvieron un porcentaje de preñez
Tabla 1. Porcentaje de preñez según tratamiento, condición corporal y estructura ovárica predominante, en vacas con cría que recibieron un tratamiento de sincronización de la ovulación e IATF.

\begin{tabular}{lc}
\hline efectos principales & $\%$ de preñez \\
\hline tratamiento & \\
$\quad$ eCG & $48,6(54 / 111)$ \\
$\quad$ control & $45,5(51 / 112)$ \\
condición corporal & \\
4 & $37,0(17 / 46)$ \\
$5-6$ & $49,7(88 / 177)$ \\
estructura ovárica predominante & \\
$\mathrm{F}<10 \mathrm{~mm}$ & $45,8(27 / 59)$ \\
$\mathrm{F} \geq 10 \mathrm{~mm}$ & $47,6(78 / 164)$ \\
\hline
\end{tabular}

de $59,3 \%$; 12,2 puntos porcentuales por encima del porcentaje de preñez observado en los animales que solo tuvieron un folículo como estructura ovárica predominante. Al analizar estadísticamente estos datos, las diferencias no son significativas; sin embargo, el bajo número de animales con cuerpo lúteo podrían explicar este hecho.

La condición corporal es uno de los principales factores que afectan el resultado de una IATF ${ }^{13}$. Así, en otro ensayo, los animales con una condición corporal de 2,5 (escala de 1 a 5; equivalente a una CC 4 de la escala 1 a 9), se preñaron en menor proporción que aquellos con una condición corporal mayor ${ }^{13}$. Sin embargo, en el presente trabajo el porcentaje de preñez de las vacas con condición corporal de 4 no difirió estadísticamente de aquéllas que tuvieron una condición corporal mayor, esto podría deberse al bajo número de animales que tuvieron una condición corporal de 4 . Por otra parte, el uso de eCG ha mejorado el porcentaje de preñez cuando se lo utiliza en animales con pobre condición corporal ${ }^{14}$, efecto que no fue observado en el presente trabajo.

Con respecto a la experiencia del técnico inseminador, se afirma que éste juega un papel preponderante en el resultado de una IATF ${ }^{13}$. En el presente trabajo se observaron diferencias significativas en el porcentaje de preñez logrado por cada inseminador, confirmando la importancia de este factor al momento de implementar una IATF. Sin embargo existe información en la que no se han observado tales diferencias ${ }^{1}$; indudablemente, la experiencia e idoneidad de los técnicos evaluados en cada caso explica las diferencias observadas.

Se concluye que el uso de la eCG, administrada al final de un tratamiento de sincronización de la ovulación basado en el uso de dispositivos intravaginales con progesterona, en vacas con cría que tienen una condición corporal de 4 a 6 y presencia de folículos como estructura ovárica predominante, no mejora el porcentaje de preñez luego de una IATF. Por el contrario, el técnico inseminador, es un factor importante que afecta la eficiencia reproductiva. 


\section{REFERENCIAS}

1. Amirat L, Bencharif D, Vera O, Pineau S, Thorin C, Destrumelle S, Desherces S, Anton M, Jouan M, Shmitt E, Tainturier D. 2010. In vivo fertility of bull semen following cryopreservation with an LDL (low density lipoprotein) extender: Preliminary results of artificial inseminations. Anim Reprod Sci 122: 282-287.

2. Bó GA, Adams GP, Pierson RA, Mapletoft RJ. 1995. Exogenous control of follicular wave emergence in cattle. Theriogenology 43: 31-40.

3. Bó GA, Baruselli OS, Moreno D, Cutaia L, Caccia M, Tríbulo R. 2002. The control of follicular wave development for self appointed embryo transfer programs in cattle. Theriogenology 57: 53-72.

4. Bó GA, Baruselli OS, Martínez MF. 2003. Pattern and manipulation of follicular development in Bos indicus cattle. Anim Reprod Sci 78: 307-326.

5. Burke CR, Mussard ML, Gasser CL, Grum DE, Day ML. 2003. Estradiol benzoate delays new follicular wave emergence in a dose-dependent manner after ablation of the dominant ovarian follicle in cattle. Theriogenology 60 : 647-658.

6. Colazo MG, Kastelic JP, Whittaker PR, Gavaga QA, Wilde R, Mapletoft RJ. 2004. Fertility in beef cattle given a new or previously used CIDR insert and estradiol, with or without progesterone. Anim Reprod Sci 81: 25-34.

7. Lamb GC, Stevenson JS, Kesler DJ, Garverick HA, Brown DR, Salfen BE. 2001. Inclusion of an intravaginal progesterone insert plus GnRH and prostaglandin F $2 \alpha$ for ovulation control in postpartum suckled beef cows. $J$ Anim Sci 79: 2253-2259.

8. Lane EA, Austin EJ, Roche JF, Crowe MA. 2001. The effect of estradiol benzoate on synchrony of estrus and fertility in cattle after removal of a progesterone-releasing intravaginal device. Theriogenology 55: 1807-1818.

9. Lane EA, Austin EJ, Roche JF, Crowe MA. 2001. The effect of estradiol benzoate or a synthetic gonadotropin- releasing hormone used at the start of a progesterone treatment on estrous response in cattle. Theriogenology 56: 7990.

10. Meneghetti M, Sá Filho OG, Peres RF, Lamb GC, Vasconcelos JL. 2009. Fixed-time artificial insemination with estradiol and progesterone for Bos indicus cows I: Basis for development of protocols. Theriogenology 72 : 179-189.

11. Perry GA, Smith MF, Lucy MC, Green JA, Parks TE, MacNeil MD, Roberts AJ, Geary TW. 2005. Relationship between follicle size at insemination and pregnancy success. Proc Natl Acad Sci USA 102: 5268-5273.

12. Rodríguez Aguilar S, Vater A, Lima D, Cabodevila J, Callejas S. 2009. Porcentaje de preñez de vacas IATF según estructura ovárica predominante al inicio de un tratamiento de sincronización de la ovulación. VIII Simposio Internacional de Reproducción Animal. IRAC (CD) Córdoba (Argentina).

13. Sá Filho OG, Meneghetti M, Peres RF, Lamb GC, Vasconcelos JL. 2009. Fixed-time artificial insemination with estradiol and progesterone for Bos indicus cows II: Strategies and factors affecting fertility. Theriogenology 72: 210-218.

14. Sá Filho MF, Ayres H, Ferreira RM, Marquez MO, Reis EL, Silva RC, Rodrigues CA, Madureira EH, Bó GA, Baruselli PS. 2010. Equine chorionic gonadotropin and gonadotropin-releasing hormone enhance fertility in a norgestomet-based, timed artificial insemination protocol in suckled Nelore (Bos indicus) cows. Theriogenology 73 : 651-658.

15. SAS. 1998. Institute Inc., SAS/STAT ${ }^{\circledR}$ User's Guide, Version 6, vol. 2., 4th ed. SAS Institute Inc., Cary, NC, 846 p.

16. Vasconcelos JL, Sartori R, Oliveira HN, Guenther JG, Wiltbank MC. 2001. Reduction in size of the ovulatory follicle reduces subsequent luteal size and pregnancy rate. Theriogenology 56: 307-314. 